\title{
Models of earth-tide-induced groundwater oscillations in the Police Basin, Czech Republic
}

\author{
T. Ondovcin ${ }^{1}$, J. Mls ${ }^{1} \&$ L. Herrmann ${ }^{2}$ \\ ${ }^{I}$ Charles University, Czech Republic \\ ${ }^{2}$ Czech Technical University, Czech Republic
}

\begin{abstract}
Earth-tide induced oscillations of hydraulic head occurring in a confined aquifer in an isolated block of rocks in the Police Basin, Czech Republic were studied. Two models were developed to demonstrate how earth-tide induced groundwater oscillations originate in the area. Due to the nature of the process, it was useful to utilize Biot's theory to predict the tidal oscillations. The resulting theory was applied in connection with two conceptual models involving geological structure of the site. This paper presents a sensitivity study of these models.
\end{abstract}

Keywords: porous media, fluid mechanics, groundwater flow, earth-tide, mathematical modeling.

\section{Introduction}

Earth is subjected to tidal force - a secondary effect of gravity of other celestial bodies, almost exclusively the Moon and the Sun. As such, tidal force acts on all matter composing Earth.

The effect tidal force has on the solid part of Earth causes stress in Earth's crust. The stress changes can affect the timing of seismic and volcanic events, e.g. [1], water flow inside mountain glaciers and in their vicinity [2] and various other phenomena. Earth-tide induced oscillations of groundwater have been known since 19th century [3] and have been observed all over the world under various geological conditions (see Melchior [4]). Melchior's simple estimate of amplitude of tidal oscillations of groundwater shows that local geology greatly influences them.

The earth-tide induced oscillations were investigated mostly in association with confined aquifers (e.g. [5-8]), partly in an effort to use wells as indicators of 
crustal strain (e.g. [9-11]). Most of these works assume an infinite confined aquifer without consideration of its constraints or position with respect to surrounding geological bodies and without taking into account distinct mechanical properties of aquifer surroundings. Work of Gieske and de Vries [12] is an exception - the authors investigated a measurement of tidal oscillations in three wells near Gaborone in Africa which were drilled into several geological bodies. The amplitudes of tidal oscillations varied depending on geological body. The authors explained this by different mechanical properties of the geological bodies. A weaker, heavily fractured formation was surrounded by hardrocks. Thus it deformed significantly more than its surroundings and this was the cause of a much larger amplitude of tidal oscillations in one well in comparison with other wells in vicinity. Thus they showed that tidal deformation or strain is not uniform on macroscale and that both amplitude and phase shift of tidal oscillations depend on geological structure as well as mechanical properties of the rocks. Well casing can also affect the tidal oscillations [13].

\section{Mathematical model}

The Police Basin comprises of several layers of various cretaceous sediments (from clayey limestone to coarse sandstones). It was fractured into several blocks during Tertiary. Well V-34 located in a narrow block of the Basin manifests tidal oscillations while other wells around, which are drilled into surrounding blocks, do not. All wells tap a thin (10 m thickness) and extremely fractured chert aquifer confined with a semipermeable layer (aquitard). It is present in the whole extent of the Basin and due to intensity of the fracturing, it is very permeable (hydraulic conductivity of order $10^{-3} \mathrm{~m} . \mathrm{s}^{-1}$ ) and considered the prime groundwater resource in the area.

The process of translation of tidal strain into groundwater oscillations is basically a mechanical interaction between the solid and the liquid phase. Biot [14] presented the first three dimensional linear theory of poroelasticity; it assumes incompressible phases, it is considered stress produces a change of porosity. Rice and Cleary [15] introduced compressibility of both phases. Since the deformation is very small and slow and the process is considered to be drained, Biot's (1941) theory was rearranged and utilized in [16] in the form

$$
\begin{gathered}
\mu \Delta u_{i}+\frac{\mu}{1-2 \nu} \frac{\partial}{\partial x_{i}}\left(\frac{\partial u_{j}}{\partial x_{j}}\right)-\alpha \frac{\partial p}{\partial x_{i}}=0, \quad i=1,2,3, \\
\alpha \frac{\partial}{\partial t}\left(\frac{\partial u_{j}}{\partial x_{j}}\right)+\left(\frac{1}{R}-\frac{\alpha}{H}\right) \frac{\partial p}{\partial t}=\frac{k}{\eta} \triangle p+q .
\end{gathered}
$$

where $x_{i}$ are the space coordinates, $t$ is time, $\mu$ is the shear modulus of the solid phase, $\nu$ is Poisson ratio of the solid phase, $u_{i}$ are components of the solid-phase displacement vector, $p$ is liquid-phase pressure, $k$ is permeability of the liquid phase, $\eta$ its dynamic viscosity and $q$ is a source term. $H$ and $R$ are coefficients 
which [14] introduced to describe the solid-liquid interaction: $1 / H$ is a measure of compressibility of the solid phase for a change in water pressure and $1 / R$ is a measure of the change in water content for a change in water pressure. Coefficient $\alpha$ is defined

$$
\alpha=\frac{2 \mu(1+\nu)}{3 H(1-2 \nu)} .
$$

The geometry of the block and its aquifer part allowed to reduce the problem to a one-dimensional case and rearrange (1) and (2) into

$$
\frac{\mathrm{d} y}{\mathrm{~d} t}=\frac{2 \mu(1-\nu)}{(1-2 \nu)} \frac{\widehat{k}}{\eta Z \widehat{Z}}\left[-y+\frac{T}{\rho_{w} g}+\widehat{h}+\widehat{Z}\right],
$$

where $Z$ is the thickness of the chert aquifer, $\widehat{k}$ is the permeability of confining layers, $\widehat{Z}$ their thickness, $\widehat{h}$ hydraulic head on the other side of the aquitard and

$$
y=h+\frac{T}{\rho_{w} g},
$$

$h$ being hydraulic head in the chert aquifer, $\rho_{w}$ density of liquid phase, $g$ the acceleration of gravity and $T$ representing the tidal stress translated through boundary from the surrounding blocks. The initial condition reads

$$
y(0)=h(0)+\frac{T(0)}{\rho_{w} g},
$$

which means one has to know the hydraulic head and tidal stress in the aquifer at a given time. Two conceptual models were presented in [16] to predict the parameters of tidal oscillations. Each prescribes a special form of the function $T$. One model links the origin of tidal oscillations to the horizontal components of tidal force in the area (see Fig. 1). The function $T$ is defined as

$$
T(t)=-M_{N}\left(\gamma_{1}(t) n_{1}+\gamma_{2}(t) n_{2}\right),
$$

where $M_{N}$ is a constant of proportionality between the tidal acceleration $\gamma, \gamma_{1}$ and $\gamma_{2}$ are horizontal components of the tidal acceleration and $n_{i}$ are components of unit vector normal to the boundary. The other model presumes the oscillations arise due to the vertical component of the tidal force (see Fig. 2). In such case

$$
T(t)=M_{N} \gamma_{3}(t)
$$

where $\gamma_{3}$ is the vertical component of the tidal acceleration.

The tidal force was computed using the tidal potential catalogue HW95 of [18]. See [16] for a detailed development of the forward problem. 


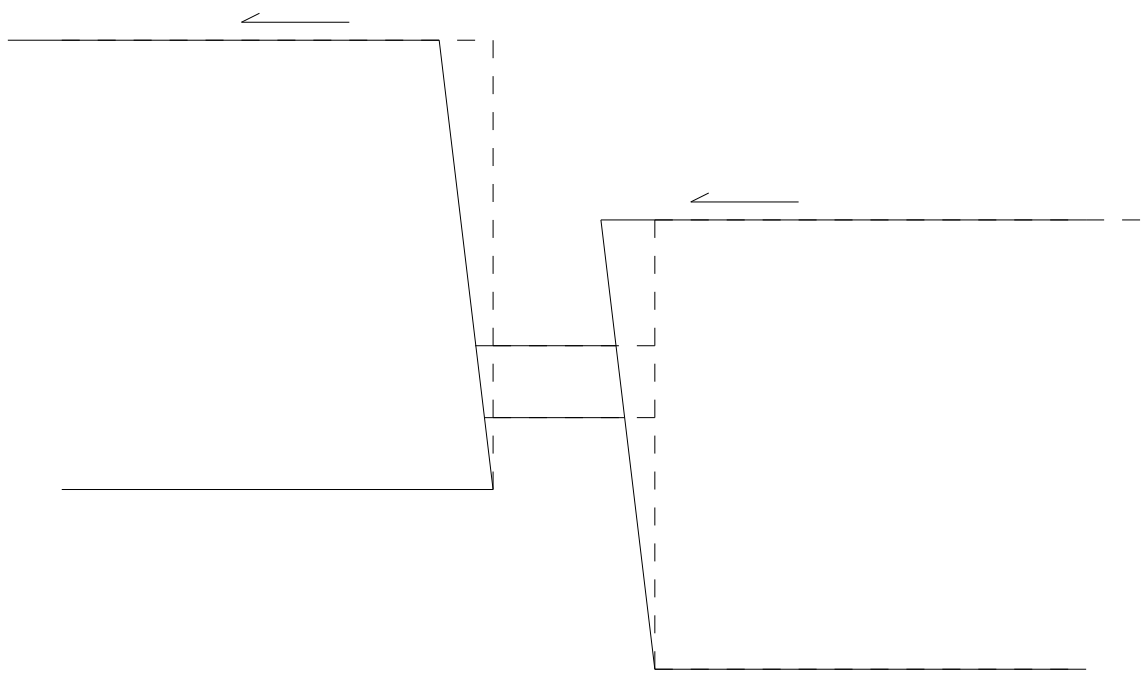

Figure 1: The concept of block movement where tidal oscillations arise due to horizontal components of the tidal force (model for horizontal components). From [17].

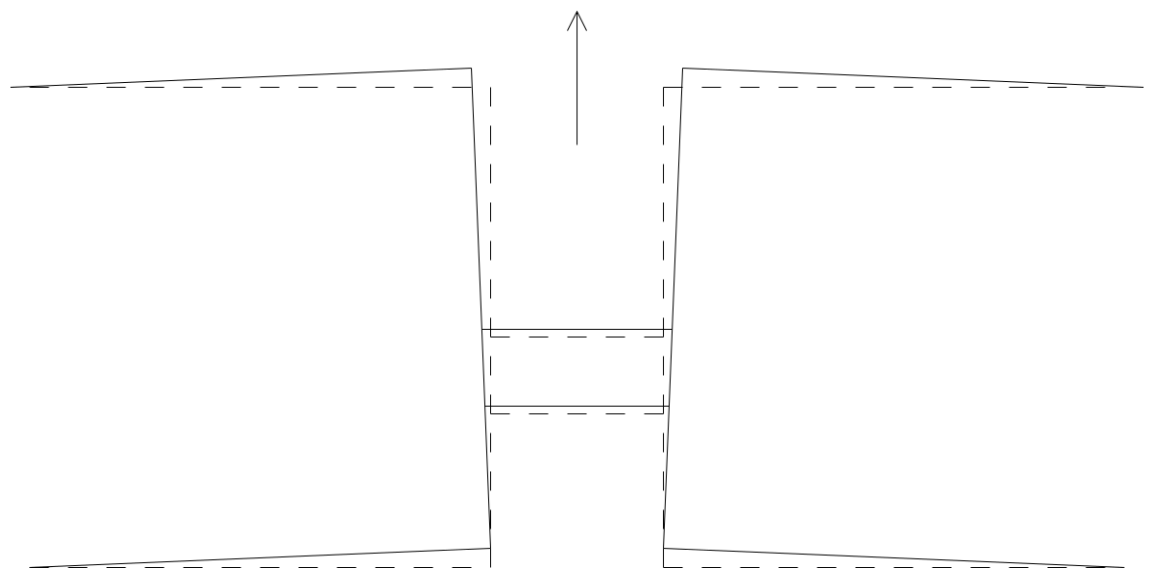

Figure 2: The concept of block movement there the vertical component of tidal force causes oscillations of hydraulic head (model for vertical component). From [17]. 


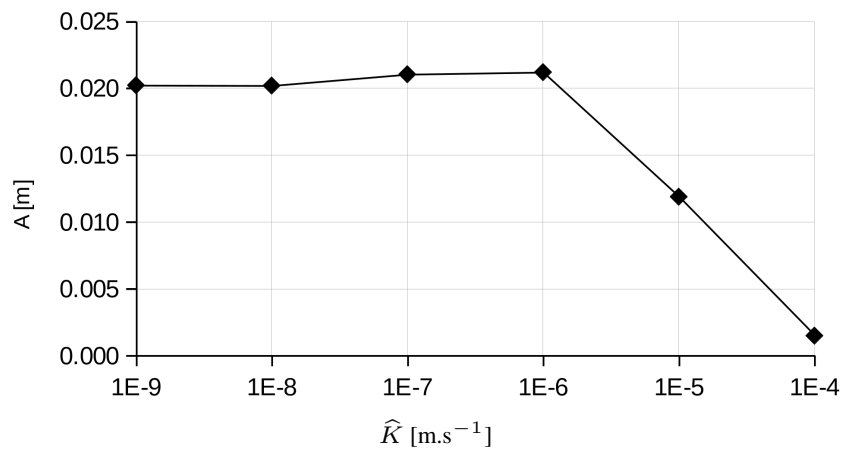

Figure 3: Relation between aquitard hydraulic conductivity $\widehat{K}$ and tidal oscillation amplitude $A-$ model for horizontal components of tidal force. From [17].

\section{Sensitivity analysis}

The forward problem has following parameters: hydraulic conductivity $\widehat{K}$ of the aquitard, thickness of the aquitard $\widehat{Z}$, aquifer thickness $Z$, shear modulus $\mu$ and Poisson ratio $\nu$ of the block and the coefficient $M_{N}$. For the purposes of sensitivity analysis, the forward problem was solved for multiple values of one of the parameters, while the others were fixed. As stated in [16], values of $\widehat{K}, \mu$ and $M_{N}$ were the most influencing.

Low hydraulic conductivity $\widehat{K}$ of the aquitard does not affect the amplitude of tidal oscillations. Values higher than $10^{-6} \mathrm{~m} . \mathrm{s}^{-1}$ decrease the amplitude (see Fig. 3).

Similarly, hydraulic conductivity affects the phase shift of tidal oscillations only for high enough values (see Fig. 4). Zero phase shift denotes no delay between tidal force and groundwater oscillations. The aquitard can account for up to a three hour delay of groundwater oscillations in a well confined aquifer. The model for horizontal component behaves almost identically, except the amplitude for low values of $\widehat{K}$ is $0.018 \mathrm{~m}$, which is slightly less than shown by the model for horizontal components.

The results of the forward problem shown in Fig. 5 demonstrate the influence hydraulic conductivity has on overall character of the oscillations. As the value changes, some local extremes at the beginning of the solution become less prominent or even vanish.

Greater thickness of the aquitard also lowers the amplitude. However, the size of the domain and geological data in [19] suggest that the thickness is uniform and therefore quite precisely defined throughout the domain. Varying it several orders of magnitude has little meaning. Depending on the aquitard thickness, amplitude of the tidal oscillations can vary in range of about $1 \mathrm{~cm}$ (see Fig. 6). The same applies 


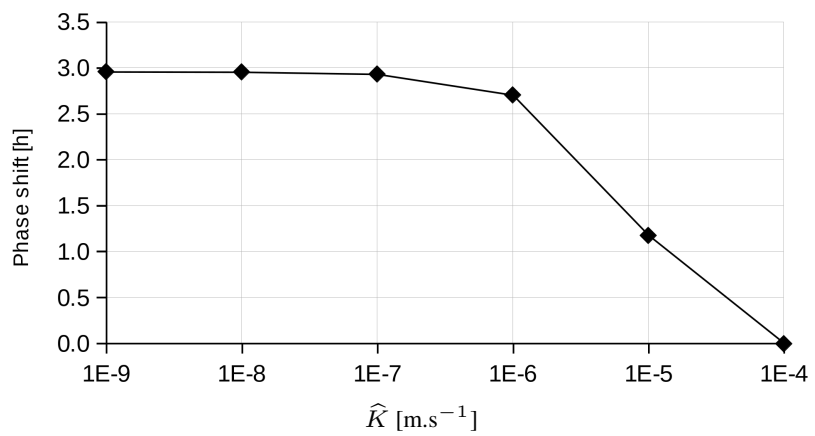

Figure 4: Relation between aquitard hydraulic conductivity $\widehat{K}$ and phase shift, i.e. delay between the tidal force and the tidal oscillations - model for horizontal components of tidal force. From [17].
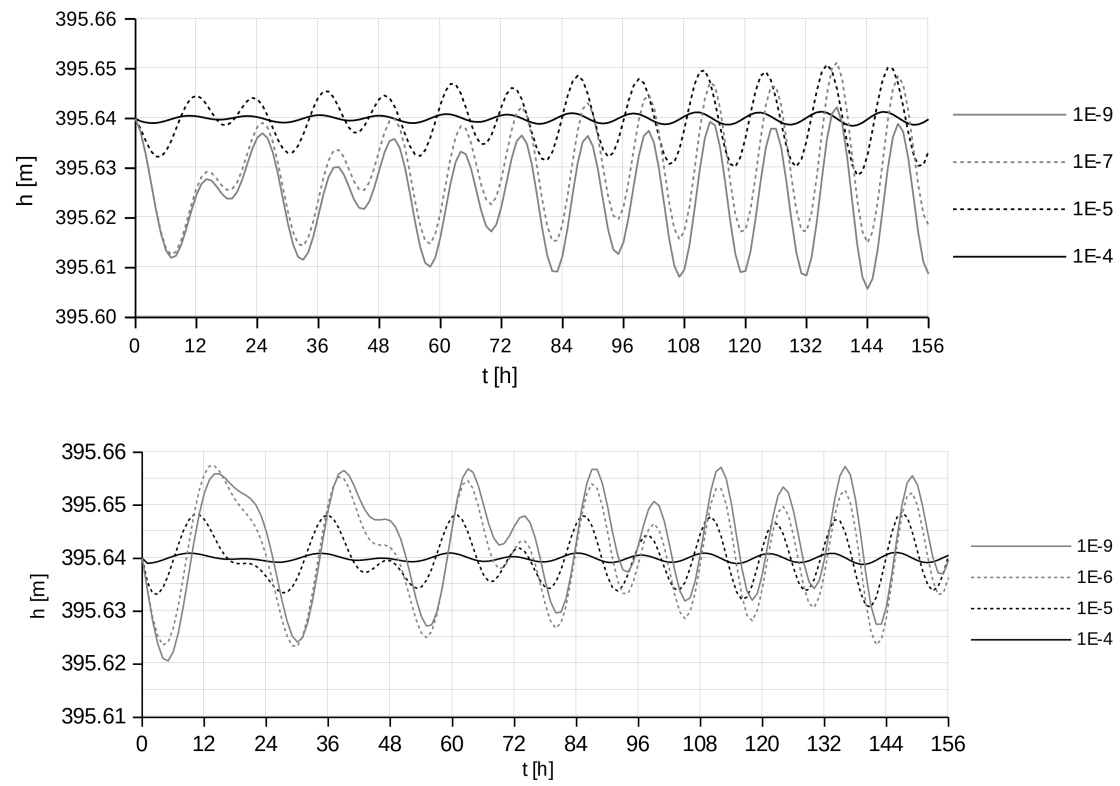

Figure 5: Tidal oscillations calculated with the model for horizontal components (top) and the model for vertical component (bottom) for several values of aquitard conductivity $\widehat{K}$ (in $\mathrm{m} \cdot \mathrm{s}^{-1}$ ). From [17]. 


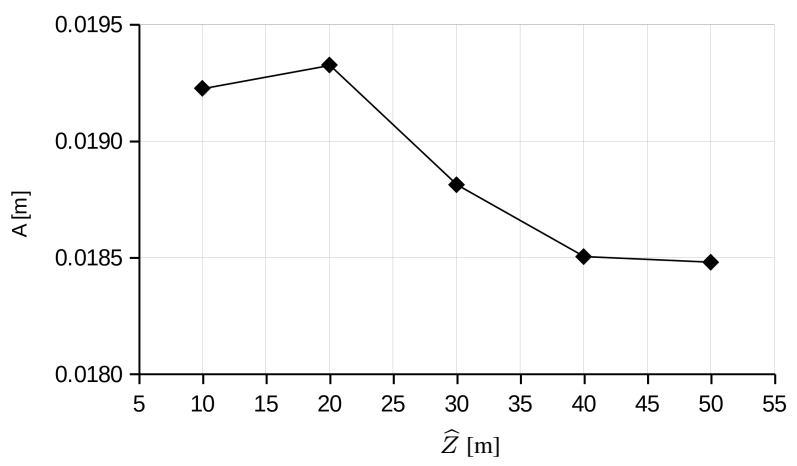

Figure 6: Amplitude of tidal oscillations depending on aquitard thickness $\widehat{Z}$ model for horizontal components of tidal force. From [17].

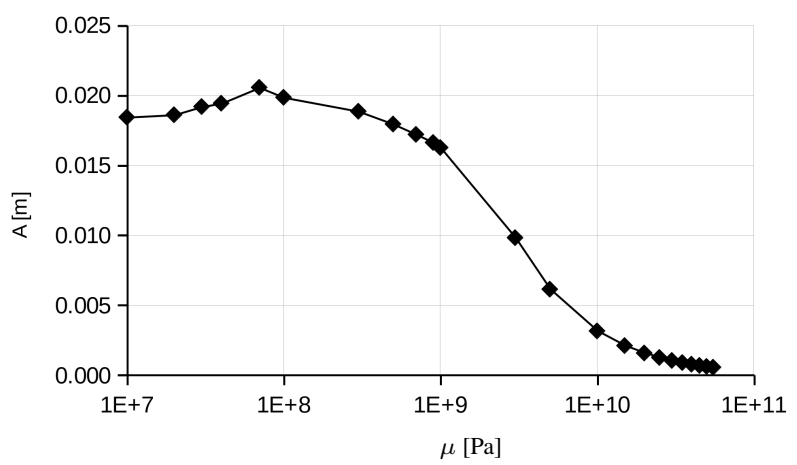

Figure 7: Amplitude of tidal oscillations depending on aquitard thickness $\widehat{Z}$ model for horizontal components of tidal force. From [17].

to the aquifer thickness $Z$ regardless of the conceptual model. The model for vertical component again predicts smaller amplitudes than the model for horizontal components.

The range of reasonable values is quite narrow for Poisson ratio $\nu$ as well. Its effects is even smaller than that of aquitard thickness - the amplitude varies within less than $1 \mathrm{~mm}$.

Shear modulus $\mu$ affects the amplitude of tidal oscillations similarly to hydraulic conductivity. Amplitude is smaller for greater values (see Fig. 7), a change in overall character of the oscillations is observable too. In the model for vertical component of tidal force, the change of shear modulus produces more pronounced change of the overall shape of the oscillations (see Fig. 8). It is also apparent that a change of the shear modulus causes the phase shift. Increasing $\mu$ two orders of magnitude decreases the phase shift by less than one hour. 

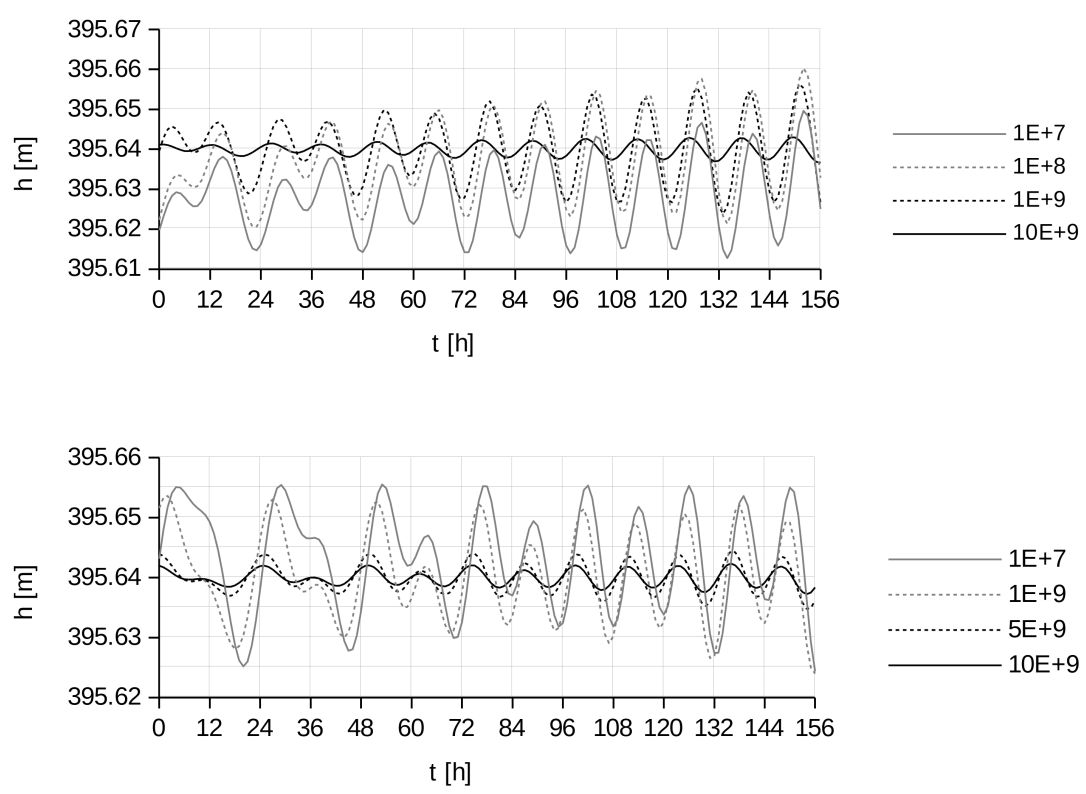

Figure 8: Tidal oscillations calculated with the model for horizontal components (top) and the model for vertical component (bottom) for several values of shear modulus $\mu$ (in Pa). From [17].

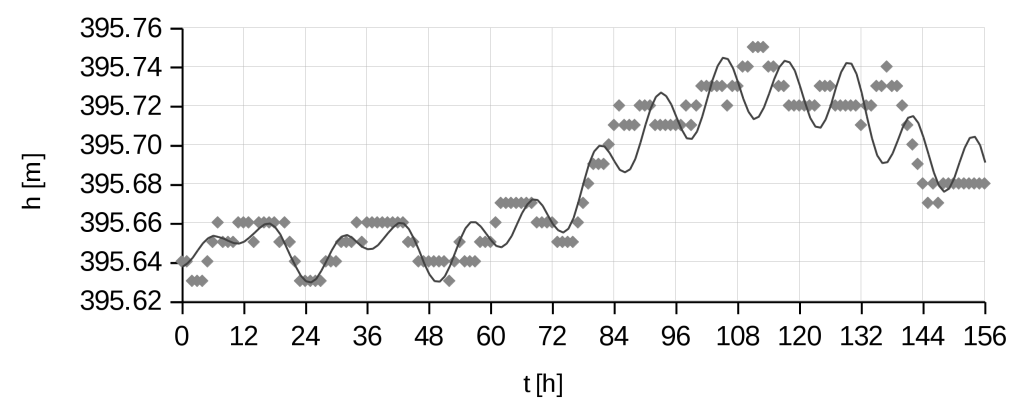

Figure 9: Comparison of the numerical solution (solid line) obtained with model for vertical component of tidal force and the measurement in V-34 (squares). From [17].

The amplitude of tidal oscillations is linearly proportional to the value of $M_{N}$. The only parameters which produce a phase shift are the hydraulic conductivity of the aquitard and the shear modulus. They both influence amplitude and overall character of the oscillations as well. 


\section{Conclusions}

Comparison between the measurement in Police Basin in [19] and prediction from theory of [16] shows that the theory produces realistic oscillations in terms of their amplitude and overall character (see Fig. 9). However, it does not perform as well concerning phase shifts. The range of possible phase shifts as shown in previous section is not as wide as exhibited in the measurement, even for a very wide range of hydraulic conductivity and shear modulus. The data also displays a non-constant phase shift. Work of [20], which was also done in Police Basin, shows that the phase shift indeed changes in time and suggested this phenomenon is related to the mechanical state of the geological formations. They noted the phase shift changes in step-wise fashion during each seismic event in the area. This is in accordance with other observations (e.g. [21]).

\section{Acknowledgement}

This work was supported with project MSM0021620855 of Ministry of Education, Youth and Sports of Czech Republic.

\section{References}

[1] Métivier, L., de Viron, O., Conrad, C.P., Renault, S., Diament, M. \& Pataub, G., Evidence of earthquake triggering by the solid Earth tides. Earth and Planetary Science Letters, 278(3-4), pp. 370-375, 2009.

[2] Kulessa, B., Hubbard, B., Brown, G.H. \& Becker, J., Earth tide forcing of glacier drainage. Geoph Res Lett, 30(1), 2003.

[3] Klönne, F.W., Die periodischen Schwankungen des Wasserspiegels in den inundirten Kohlenschächten von Dux in der Periode vom 8. April bis 15 . September 1879. Akad Wiss Wien Sitzungsber, LXXXI, pp. 114-116, 1880.

[4] Melchior, P., The tides of the planet Earth. Pergamon press, 1983.

[5] Bredehoeft, J.D., Response of well-aquifer systems to Earth tides. J Geoph Res, 72(12), pp. 3075-3087, 1967.

[6] Robinson, E.S. \& Bell, R.T., Tides in confined well-aquifer systems. J Geoph Res, 76(8), pp. 1857-1869, 1971.

[7] Ritzi, R.W., Sorooshian, S. \& Hsieh, P.A., The estimation of fluid-flow properties from the response of water levels in wells to the combined atmospheric and Earth tide forces. Water Resour Res, 27(5), pp. 883-893, 1991.

[8] Rojstaczer, S. \& Agnew, D.C., The influence of formation material properties on the response of water levels in wells to Earth tides and atmospheric loading. J Geoph Res, 94(B9), pp. 12403-12411, 1989.

[9] Bodvarsson, G., Confined fluids as strain meters. J Geoph Res, 75(14), pp. 2711-2718, 1970. 
[10] Lewkowicz, J., Tilt observations from shallow and deep borehole arrays. Phys of The Earth and Planetary Interiors, 41(1), pp. 58-65, 1985.

[11] Zeumann, S., Weise, A. \& Jahr, T., Tidal and non-tidal signals in groundwater boreholes in the KTB area, Germany. Journal Of Geodynamics, 48(3-5, Sp. Iss. SI), pp. 115-119, 2009. 16th International Symposium on Earth Tides, Jena, Germany, SEP 01-05, 2008.

[12] Gieske, A. \& de Vries, J.J., An analysis of earth-tide-induced groundwater flow in eastern Botswana. J Hydrol, 82, pp. 211-232, 1985.

[13] Hsieh, P.A., Bredehoeft, J.D. \& Farr, J.M., Determination of aquifer transmissivity from Earth tide analysis. Water Resour Res, 23(10), pp. 1824 1832, 1987.

[14] Biot, M.A., General theory of three-dimensional consolidation. J Appl Phys, 12, pp. 155-164, 1941.

[15] Rice, J.R. \& Cleary, M.P., Some basic stress diffusion solutions for fluidsaturated porous media with compressible constituents. Rev Geophys Space Phys, 14, pp. 227-241, 1976.

[16] Ondovčin, T. \& Mls, J., Mathematical modeling of tidal effects in groundwater. Transp Porous Med, 93(3), 2012. DOI 10.1007/s11242-0120056-3.

[17] Ondovčin, T., Tidal phenomena in groundwater hydraulics. Dissertation, Charles University, Faculty of Science, 2012.

[18] Hartmann, T. \& Wenzel, H.G., The HW95 tidal potential catalogue. Geophys Res Lett, 22, pp. 3353-3556, 1995.

[19] Krásný, J., Buchtele, J., Čech, S., Hrkal, Z., Jakeš, P., Kobr, M., Mls, J., Šantrůček, J., Šilar, J. \& Valečka, J., Hydrogeology of the Police Basin: Optimisation of groundwater development and protection. J Geol Sci, 22, pp. 5-100, 2002.

[20] Kolínský, P., Valenta, J. \& Gaždová, R., Seismicity, groundwater level variations and earth tides in the Hronov-Poříćí fault zone, Czech Republic. Acta Geodyn Geomater, 9(2 (166)), pp. 191-209, 2012.

[21] Elkhouri, J.E., Brodsky, E.E. \& Agnew, D.C., Seismic waves increase permeability. Nature, 411, pp. 1135-1138, 2006. 